\title{
FUNCTIONAL AND DETERMINISTIC TUNING OF HYBRID INTEGRATED ACTIVE FILTERS
}

\author{
G. S. MOSCHYTZ \\ Swiss Federal Institute of Technology, Zurich, Switzerland
}

(Received June 15, 1977)

\begin{abstract}
The two main methods of tuning hybrid integrated active filters, namely functional and deterministic tuning are described. Functional tuning implies the fine adjustment of an active network that is assembled and in operation so that its transmission characteristics (e.g. gain or phase response) can be monitored during the tuning process. One or several resistors are selected as tuning elements and these are adjusted (e.g. by laser, anodization, sandblasting, "postbox" selection of discrete resistors) until the desired response is obtained. Deterministic tuning involves the fine adjustment of individual resistors to values which are analytically predicted. The prediction is based on network equations which include parasitic effects and in which those component values that are not to be adjusted are obtained by measurements on the (non-operational) manufactured circuit. Manufacturing tolerances and parasitic effects are included in the comprehensive set of equations whose solutions provide the final adjustment values of a few 'tuning resistors'. Accuracy bounds and limitations of the two tuning methods are given and the pros and cons of the two methods are discussed. Finally it is suggested that a well balanced combination of the two methods may well provide the most efficient and least complicated solution to the tuning problem in hybrid integrated networks.
\end{abstract}

\section{INTRODUCTION}

As active hybrid-integrated filters are increasingly being developed for modern communication systems, ${ }^{1-7}$ so the question of how to tune them to specifications most efficiently, and at minimum cost, is becoming ever more important. In practice two basically different tuning methods can be distinguished, namely functional and deterministic tuning. ${ }^{8}$

Functional tuning implies tuning the critical parameters of a network while it is functional, i.e. in operation. Because the network is assembled as for operation in the final system, any parasitics built into the network are automatically taken into account and "tuned out" during the tuning process. Functional tuning is generally iterative, particularly if the tuning steps are interactive (Figure 1). The number of iterations will increase with the degree of tuning accuracy required. The larger the number of iterative tuning steps, the more time consuming, and therefore the more costly, the tuning process will be. Functional tuning will generally be preferable for laboratory purposes and when production quantities are moderate or low.

Deterministic tuning implies tuning, or trimming to value, individual components of a network as predicted by a combination of comprehensive network equations (in which parasitic effects are taken into account) and by component measurements (Figure 2). The solutions of equations (generally obtained by an on-line computation facility) provide the values of the components to be tuned. Tuning is carried out "to value", hence it makes no difference whether the network is operational or not. Since the components to be tuned are invariably resistors, this method consists of "resistor trimming", in contrast to the tuning of network characteristics (e.g. amplitude, phase, frequency) that occurs in functional tuning. The method is simple ("to-value" trimming of resistors is essential in any hybrid integrated circuit manufacturing plant) and rapid in execution (generally very few, if any, iterations are required). However, powerful computer programs will be required to solve the nonlinear network equations which must take first, and often second order parasitic effects into account. Deterministic tuning is the more efficient of the two methods, but the necessary expenditure of an on-line computation facility, and the initial computational effort required, can generally be justified only by very high production volumes.

In practice it will very often be found useful to combine functional with deterministic tuning. The initial adjustments will be carried out by deterministic tuning, where the values are obtained from either the idealized network equations or from 


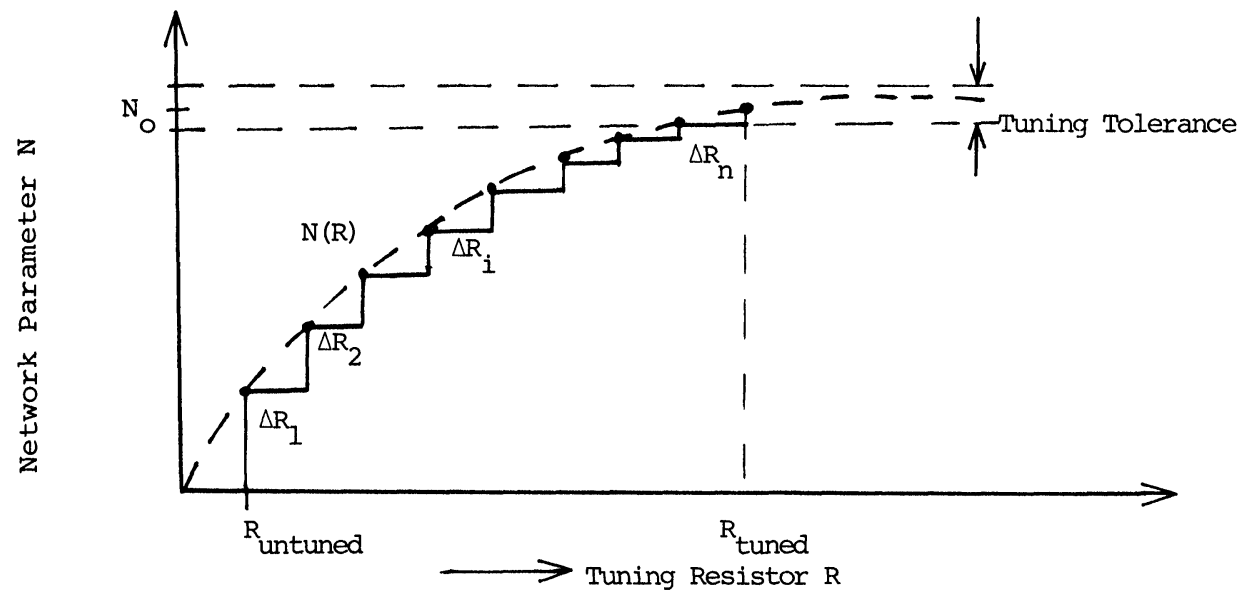

FIGURE 1 Functionally tuning to a parameter value $N_{0}$ with the tuning resistor $R$.

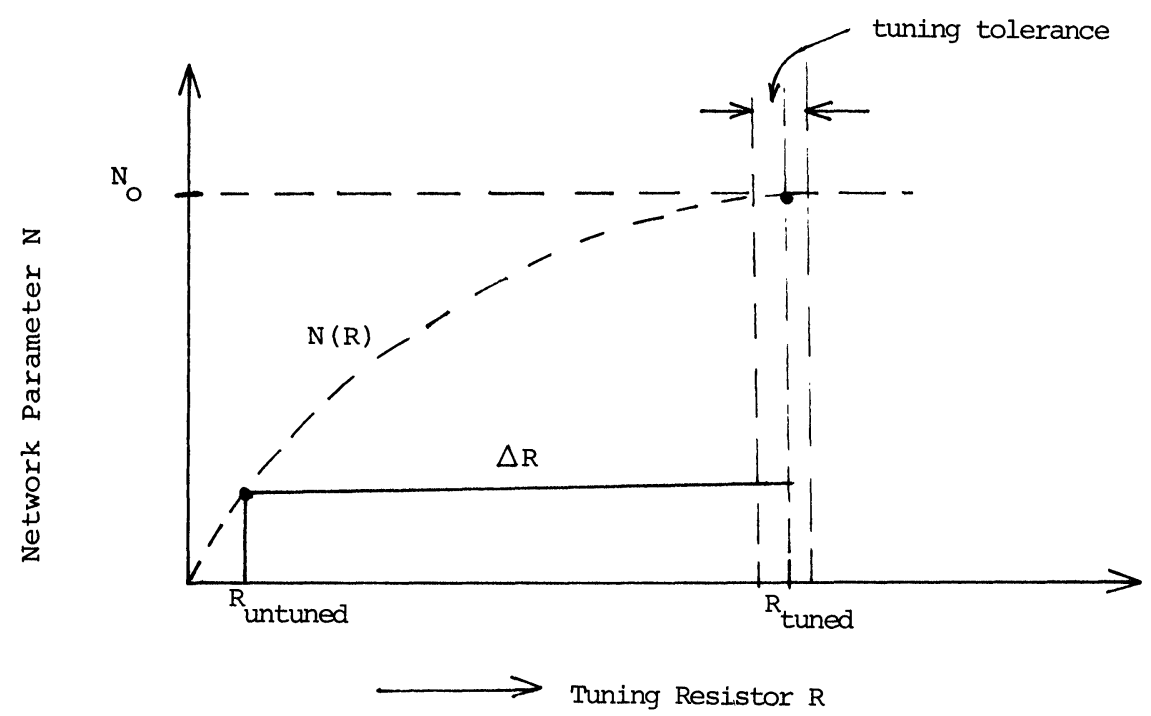

FIGURE 2 Deterministically tuning to a parameter value $N_{0}$ with the tuning resistor $R$.

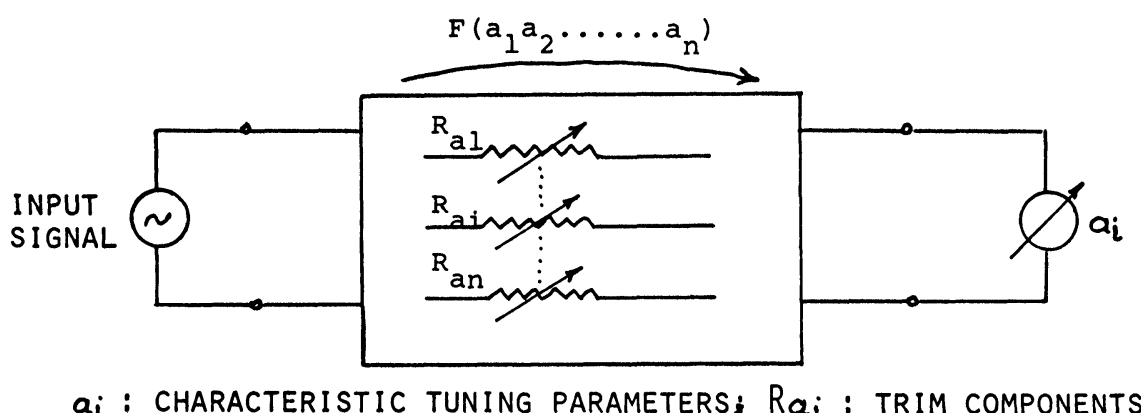

FIGURE 3 Functionally tuning the characteristic tuning parameters $a_{i}$ with the corresponding resistors $R_{a}$ to obtain the specified function $F\left(a_{i}\right)$. 
those containing at most first-order parasitic effects. To overcome more subtle second-order parasitics, a fine-tuning step is then undertaken in which the circuit is operational (i.e. assembled and powered) and a functional adjustment of one or more of the critical parameters is carried out. In this way the computational complexity inherent in deterministiconly tuning can be considerably reduced.

\section{FUNCTIONAL TUNING}

Functional tuning is based on a set of network equations in which the changes of the specified network characteristics $F_{j}, j=1,2, \ldots, m$ are related to incremental changes in the components $x_{i}, i=1$, $2, \ldots, n$, by the sensitivity matrix, hence:

$$
\left[\begin{array}{c}
\Delta F_{1} / F_{1} \\
\vdots \\
\Delta F_{m} / F_{m}
\end{array}\right]=\left[\begin{array}{ccc}
S_{x_{1}}^{F_{1}} \ldots & S_{x_{i}}^{F_{1}} \ldots & S_{x_{n}^{1}}^{F_{1}} \\
\vdots & & \vdots \\
S_{x_{1}}^{F_{m}} \ldots & \ldots S_{x_{i}}^{F_{m}} \ldots & S_{x_{n}}^{F_{m}}
\end{array}\right]\left[\begin{array}{c}
\Delta x_{1} / x_{1} \\
\vdots \\
\Delta x_{i} / x_{i} \\
\Delta x_{m} / x_{m}
\end{array}\right]
$$

where the sensitivities $S_{x_{i}}^{F_{j}}$ are defined by

$$
S_{x_{i}}^{F_{i}}=\frac{d F_{j}}{d x_{i}} \cdot \frac{x_{i}}{F_{j}}
$$

Hence, if $a_{i}(i=1, \ldots, n)$ are the characteristic tuning parameters of a network function $F\left(a_{i}\right)$ and $R a_{i}$ are the corresponding tuning resistors (Figure 3 ), then tuning the parameters from $a_{i}+\Delta a_{i}$ to $a_{i}$ will adjust the network function from its initial form $F\left(a_{i}+\Delta a_{i}\right)$ to the desired form $F\left(a_{i}\right)$. Letting

$$
S_{R a_{j}}^{a_{i}}=\frac{d a_{i} / a_{i}}{d R a_{j} / R a_{j}}
$$

we then obtain the sensitivity relations:

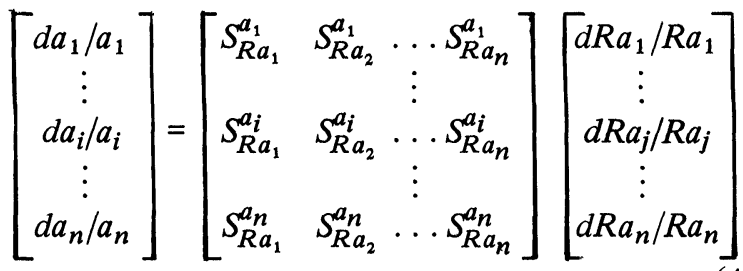

or

$$
\left[\frac{d a}{a}\right]=[S] \cdot\left[\frac{d R}{R}\right]
$$

where $[S]$ is the sensitivity matrix.

In order to allow for a noninteractive, and hence noniterative, tuning procedure the sensitivity matrix must be a diagonal matrix, meaning that all offdiagonal matrix elements must be zero (Figure 4a). In practice this will rarely be the case. However, it may be possible to arrange the sensitivity matrix such that it is triangular with the upper triangular elements being zero (Figure 4b). For each network characteristic $a_{k}$ there is then a tuning element $R a_{k}$ that leaves all previously tuned parameters $a_{j}(j<k)$ unaffected. The tuning sequence is critical; it results directly from the sensitivity matrix after the latter has been arranged in triangular form. The obtained sequence provides a single-pass (or one-shot), noninteractive tuning procedure requiring no iterations. If a triangular matrix in the form of Figure $4 b$ cannot be obtained accurately, it must be approximated by arranging the matrix elements such that they decrease in value to the right of the diagonal (Figure 4c). In this way, the number of tuning iterations can be minimized.

Consider, for example, a second-order network possessing two conjugate complex poles $p_{1,2}=$ $-\sigma+j \widetilde{\omega}$. The corresponding transfer function will have the form:

$$
T(s)=\frac{N(s)}{\left(s+p_{1}\right)\left(s+p_{2}\right)}
$$

Assuming that the two poles $p_{1}$ and $p_{2}$ are specified and must be tuned for, we now derive the matrix equation corresponding to (4) with respect to the $n$ resistors in the network. (Capacitors cannot be adjusted in hybrid-integrated circuits and are therefore not considered as tuning components.) We obtain:

$\left[\begin{array}{l}\frac{d p_{1}}{p_{1}} \\ \frac{d p_{2}}{p_{2}}\end{array}\right]=$

$$
\left[\begin{array}{cc}
u_{11} \underbrace{S_{R_{1}}^{p_{1}}} & \\
u_{21}+j v_{11} & u_{12}+j v_{12} \ldots u_{1 n}+j v_{1 n} \\
S_{R_{1}}^{p_{2}} & u_{22}+j v_{22} \ldots u_{2 n}+j v_{2 n}
\end{array}\right]\left[\begin{array}{c}
d R_{1} / R_{1} \\
\vdots \\
\vdots \\
d R_{n} / R_{n}
\end{array}\right]
$$




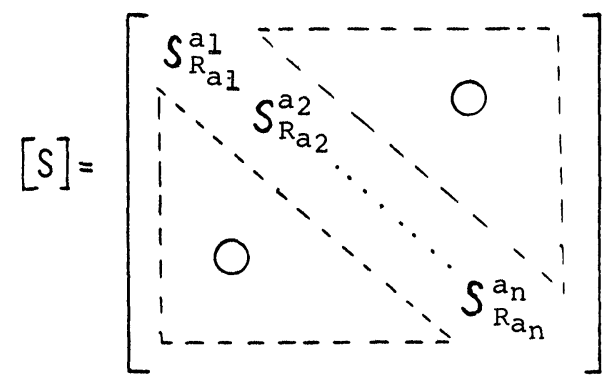

DIAGONAL MATRIX

(a)

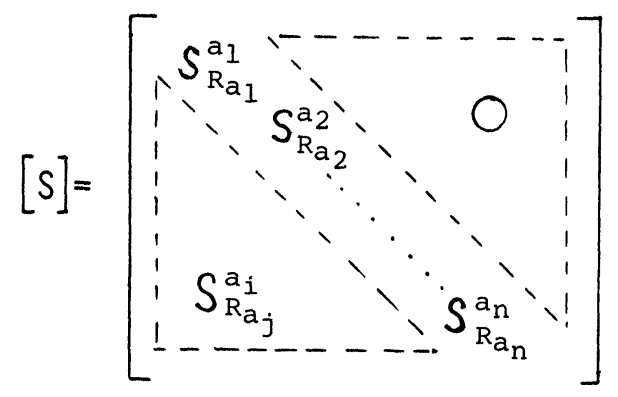

TRIANGULAR MATRIX

(b)

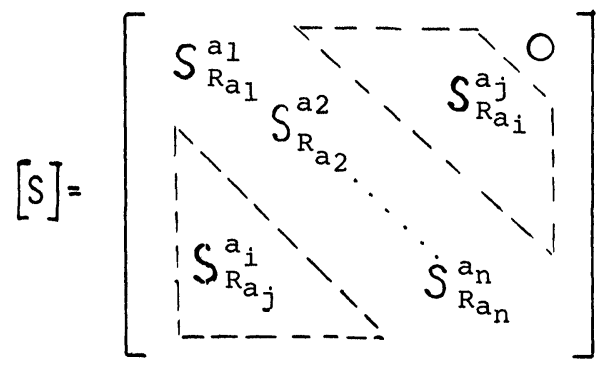

(c)

\section{ARB I TRARY NONI TERATIVE}

TUNING SEQUENCE
PRESCRIBED NONITERATIVE

TUNING SEQUENCE:

$R a_{1} \rightarrow R a_{2} \rightarrow \cdots \rightarrow R a_{i} \cdots \rightarrow R a_{n}$

\section{PRESCRIBED ITERATIVE}

TUNING SEQUENCE

$R a_{1} \rightarrow R a_{2} \rightarrow \ldots R a_{i} \rightarrow \ldots R a_{n}$
$R a_{j} \longrightarrow \ldots R a_{n}$

$$
R a_{k} \rightarrow R a_{n}
$$

FIGURE 4 Functional tuning strategies a) non-interactive b) interactive, one-pass c) general interactive.

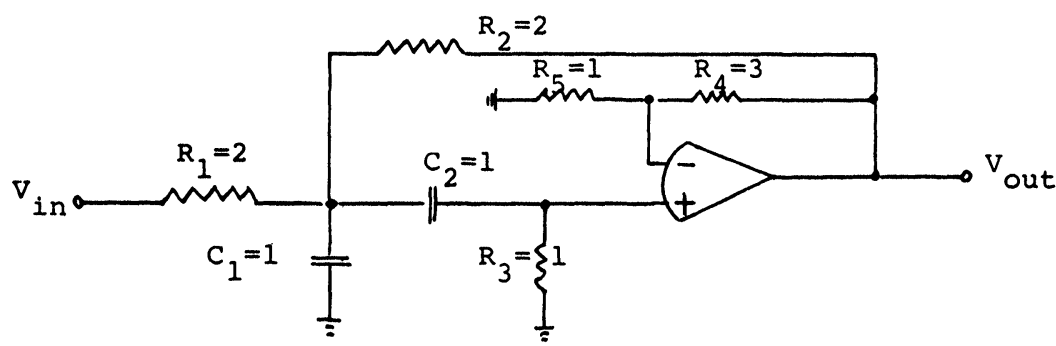

FIGURE 5 Second-order bandpass network. 
or, in matrix form:

$$
\left[\frac{d p}{p}\right]=[u+j v] \cdot\left[\frac{d R}{R}\right]
$$

It can be shown ${ }^{9}$ that:

$$
\frac{d p}{p}=\frac{d \omega_{p}}{\omega_{p}}-j \mid \frac{1}{\sqrt{4 q_{p}^{2}-1}} \cdot \frac{d q_{p}}{q_{p}}
$$

where $\omega_{p}=\sqrt{\sigma^{2}+\widetilde{\omega}^{2}}$ and $q_{p}=\omega_{p} / 2 \sigma$. Thus it follows that in (6):

$$
u_{i}=S_{R_{i}}^{\omega_{p}}
$$

and

$$
v_{i}=-\left(4 q_{p}^{2}-1\right)^{-1 / 2} \cdot S_{R_{i}}^{q_{p}}
$$

In terms of the pole frequency $\omega_{p}$ and the pole $Q$, $q_{p}$, we therefore obtain from Eq. (6a):

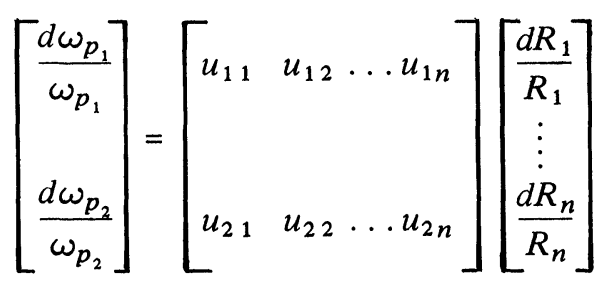

and

$\left[\begin{array}{l}\frac{d q_{p_{1}}}{q_{p_{1}}} \\ \frac{d q_{p_{2}}}{q_{p_{2}}}\end{array}\right]=-\sqrt{4 q_{p}^{2}-1}\left[\begin{array}{ccc}v_{11} & v_{12} \ldots v_{1 n} \\ v_{21} & v_{22} \ldots v_{2 n}\end{array}\right]\left[\begin{array}{l}\frac{d R_{1}}{R_{1}} \\ \frac{d R_{n}}{R_{n}}\end{array}\right]$

Since $p_{1}$ and $p_{2}$ are complex conjugate, the variations of $\omega_{p_{1}}, \omega_{p_{2}}$ and $q_{p_{1}}, q_{p_{2}}$ will not be independent of one another and we need consider only the variation $d \omega_{p} / \omega_{p}$ and $d q_{p} / q_{p}$. for a second order network.

Let us now consider the second-order active bandpass network shown in Figure 5. The voltage transfer function is

$$
T(s)=K \frac{s}{\left(s+p_{1}\right)\left(s+p_{2}\right)}=K \cdot \frac{s}{s^{2}+\frac{\omega_{p}}{q_{p}} s+\omega_{p}^{2}}
$$

In practice, $\omega_{p}$ and $q_{p}$ rather than the pole pair $p_{1}$ and $p_{2}$ will be specified, since the former are accurately measurable quantities. In addition, the constant $K$ will be specified, although in general with a much wider tolerance than $\omega_{p}$ and $q_{p}$. Deriving the sensitivity matrix with respect to the five resistors of the network, we obtain:

$$
\begin{aligned}
& {\left[\begin{array}{c}
d K / K \\
d \omega_{p} / \omega_{p} \\
d q_{p} / q_{p}
\end{array}\right]=} \\
& {\left[\begin{array}{ccccc}
-1 & 0 & 0 & 1 & -1 \\
-0.25 & -0.25 & -0.5 & 0 & 0 \\
0.25 & -1.75 & 1.5 & 1.5 & -1.5
\end{array}\right]\left[\begin{array}{l}
d R_{1} / R_{1} \\
d R_{2} / R_{2} \\
d R_{3} / R_{3} \\
d R_{4} / R_{4} \\
d R_{5} / R_{5}
\end{array}\right]}
\end{aligned}
$$

We must now rearrange the sensitivity matrix in Eq. (11) so as to provide a tuning sequence comprising three resistors and requiring a minimum number of iterations. (Clearly neither a purely diagonal nor a triangular matrix can be obtained.) The best we can do is with the resistors $R_{1}, R_{3}$ and $R_{4}$, namely:

$$
\left[\begin{array}{c}
d K / K \\
d \omega_{p} / \omega_{p} \\
d q_{p} / q_{p}
\end{array}\right]=\left[\begin{array}{ccl}
-1 & 0 & 1 \\
-0.25 & -0.5 & 0 \\
0.25 & 1.5 & 1.5
\end{array}\right]\left[\begin{array}{c}
d R_{K} / R_{K} \\
d R \omega_{p} / R \omega_{p} \\
d R q_{p} / R q_{p}
\end{array}\right]
$$

where $R_{K}=R_{1}, R_{\omega_{p}}=R_{3}$ and $R_{q_{p}}=R_{4}$. The optimum tuning sequence is then $R_{1}(K) \rightarrow R_{3}\left(\omega_{p}\right) \rightarrow$ $R_{4}\left(q_{p}\right)_{\mathcal{K}_{K}}$ whereby a slight error will be accrued in $K$ since $S_{R q_{p}}^{K} \neq 0$.

\section{ACCURACY CONSIDERATIONS FOR FUNCTIONAL TUNING}

When tuning for the frequency response of a network, we have the choice (in the case of minimum-phase networks) of tuning to a specified amplitude or to a specified phase. The question is which of these two physical quantities provide more convenient indicators for the tuning process. If we tune for an amplitude response as indicated in Figure 6a we are concerned with the variation of the amplitude $\alpha$ (e.g. in $\mathrm{dB}$ ) at a particular frequency $\omega_{i}$ with respect to the variation of a corresponding tuning resistor $R_{\alpha_{i}}$, thus:

$$
\left[d \alpha\left(\omega_{i}\right)\right]=\left[\mathcal{Y}_{R_{\alpha_{i}}}^{\alpha\left(\omega_{i}\right)}\right] \cdot\left[\frac{d R_{\alpha_{i}}}{R_{\alpha_{i}}}\right]
$$




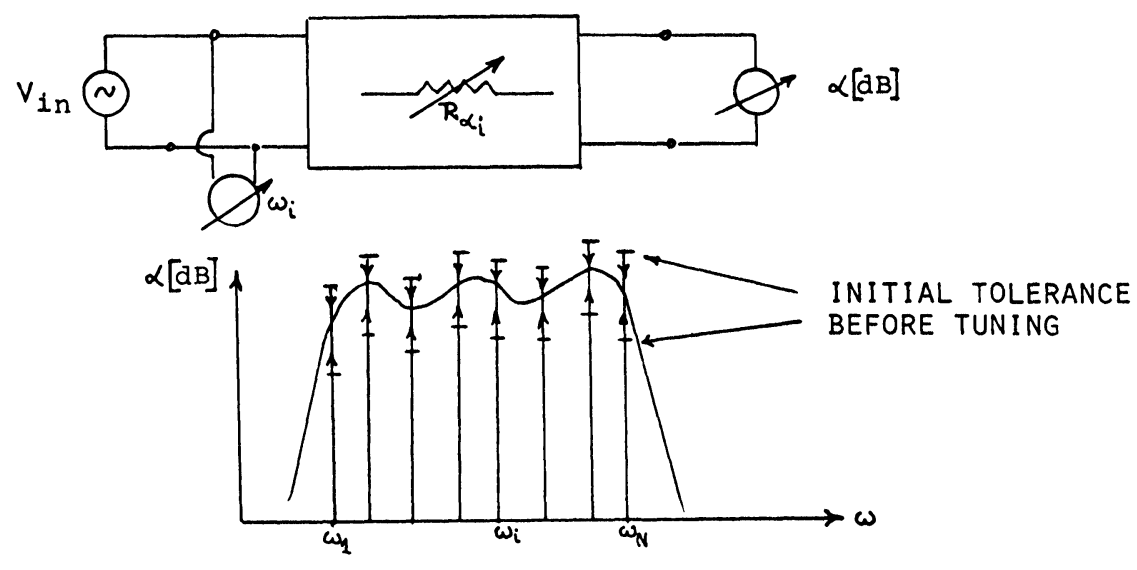

a)

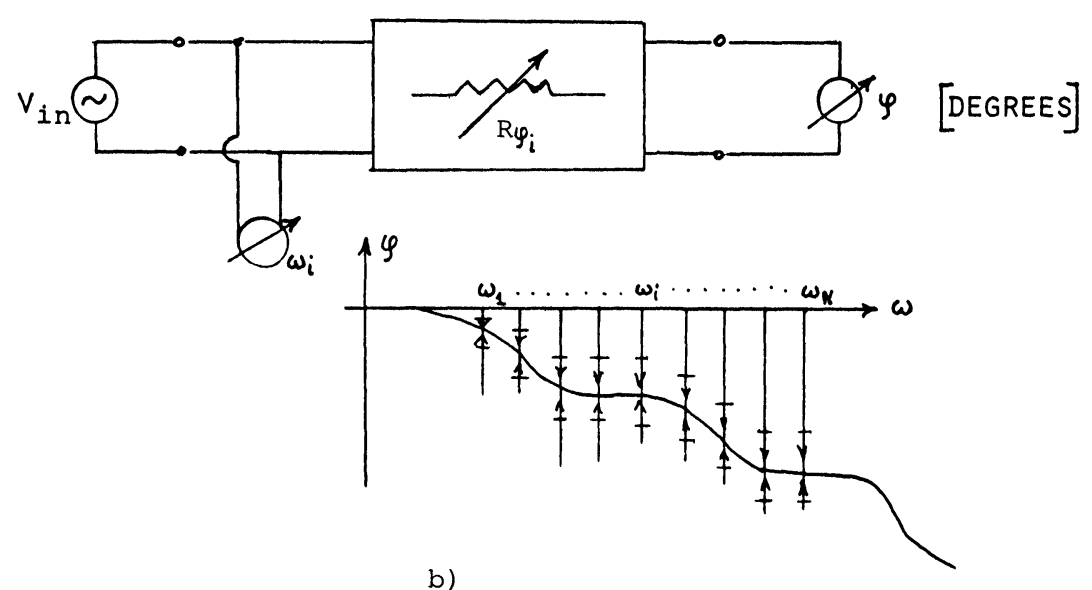

FIGURE 6 Functional tuning indicators a) amplitude b) phase.

where:

$$
\mathscr{\varphi}_{R}^{\alpha}=\frac{d \alpha}{d R / R}
$$

Similarly, tuning for a phase response (Figure $6 \mathrm{~b}$ ), the corresponding relationship between the phase $\phi\left(\omega_{i}\right)$ and the tuning resistor $R_{\phi_{i}}$ will be:

$$
\left[d \phi\left(\omega_{i}\right)\right]=\left[\mathscr{Y}_{R_{\phi_{i}}}^{\phi\left(\omega_{i}\right)}\right]\left[\frac{d R_{\phi_{i}}}{R_{\phi_{i}}}\right]
$$

where

$$
S_{R}^{\phi}=\frac{d \phi}{d R / R}
$$

At first sight it would seem that the sensitivities Eq. (12b) and Eq. (13b) will determine whether the amplitude $\alpha$ or the phase $\phi$ is a preferable tuning indicator for a given minimum-phase network. However, it can be shown ${ }^{8}$ that no matter what the network, the phase is always a more accurate indicator with which to tune the response of a network. In fact the tuning accuracy of the amplitude response of a second-order all-pole network is within $\pm 2 \Delta \phi \%$ or $\pm 0.2 \Delta \phi \mathrm{dB}$, when trimmed by means of a phase meter with an accuracy of $\pm \Delta \phi^{\circ}$. This is independent of the pole $Q$. Thus, with the attainable phase accuracy given by $\Delta \phi$ degrees, the resulting amplitude accuracy in $\mathrm{dB}$ will be:

$$
\Delta \alpha \leqslant 2 \Delta \phi \% \approx 0.2 \Delta \phi[\mathrm{dB}]
$$

It follows that a $1^{\circ}$ phase error corresponds to a $0.2 \mathrm{~dB}$ error in amplitude. Phase meters with up to 
$0.1^{\circ}$ phase accuracy are available at prices considerably lower than voltage meters with comparable accuracy (i.e. $0.02 \mathrm{~dB}$ ). It follows that wherever possible, functional tuning by phase is to be preferred over tuning by amplitude. The pole frequency error resulting from a phase error $\Delta \phi^{\circ}$ is then:

$$
\frac{\Delta f_{p}}{f_{p}}[\%]=\frac{-\pi \cdot 100}{360 q_{p}} \Delta \phi \approx-\frac{\Delta \phi}{q_{p}}
$$

where $\Delta \phi$ is measured in degrees and $q_{p}$ is the pole $Q$. Note that the higher $q_{p}$ the smaller the frequency error resulting from a given phase error. Thus, with $0.1^{\circ}$ phase error, the frequency error will be $-0.1 \%$ if $q_{p}=1$ and $-0.01 \%$ if $q_{p}=10$.

The accuracy of $q_{p}$ associated with a phase error of $\Delta \phi$ degrees can be shown to be independent of $q_{p}$. It is approximately given by:

$$
\frac{\Delta q_{p}}{q_{p}}[\%] \approx-3.5 \Delta \phi
$$

provided that the phase is measured at the polefrequency phase, plus|or|minus 45 degrees.

Consider, for example, the lowpass amplitude and phase characteristic shown in Figure 7. From the corresponding transfer function

$$
T(s)=K \frac{\omega_{p}^{2}}{s^{2}+\frac{\omega_{p}}{q_{p}} s+\omega_{p}^{2}}
$$

we obtain the amplitude response

$$
\alpha(\omega)=\ln |T(j \omega)|
$$

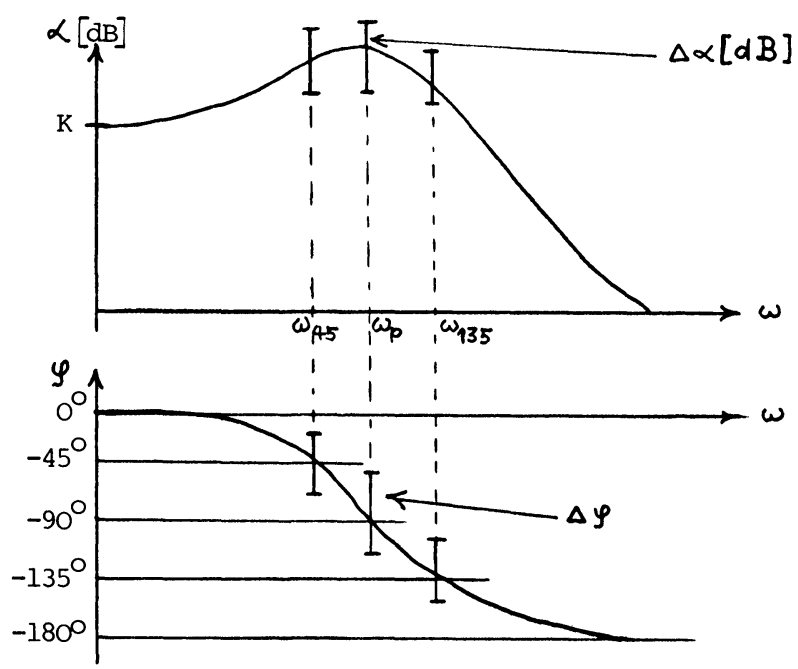

FIGURE 7 Amplitude and phase characteristic of a secondorder lowpass network. and the phase response:

$$
\phi(\omega)=\arg T(j \omega)
$$

To tune for the specified $\omega_{p}$ and $q_{p}$ values, we tune for $-90^{\circ}$ at the frequency $\omega_{p} / 2 \pi$ using an appropriate resistor $R_{\omega_{p}}$, and for $-45^{\circ}$ (or $-135^{\circ}$ ) at the frequency $\omega_{45} / 2 \pi$ (or $\omega_{135} / 2 \pi$ ) using an appropriate resistor $R_{q_{p}}$. With a phase meter accurate to within $\Delta \phi$ degrees, the errors in amplitude, frequency and $q_{p}$ will be as given by Eq. (14), (15) and (16) respectively. $K$ can also be tuned for. Remember that an error in $K$ implies an error in the dc gain of the network.

\section{DETERMINISTIC TUNING}

For a given network, the deterministic tuning procedure follows the flow-chart presented in Figure 8 . Thus for the second-order lowpass network shown in Figure 9, whose transfer function was given by Eq. (17), we proceed as follows ${ }^{8}$

1) Derive the three characteristic network parameters $K, \omega_{p}$ and $q_{p}$ as a function of the circuit components, i.e.

$$
\begin{aligned}
K & =f_{K}\left(\beta, R_{1}, R_{2}, C_{3}, C_{4}\right) \\
\omega_{p} & =f_{\omega_{p}}\left(R_{1}, R_{2}, R_{4}, C_{3}, C_{4}\right) \\
q_{p} & =f_{q_{p}}\left(\beta, R_{1}, R_{2}, R_{4}, C_{3}, C_{4}\right)
\end{aligned}
$$

2) Measure the capacitors $C_{3}, C_{4}$ and the closedloop amplifier gain $\beta$

3) Compute the following resistor values as a function of quantities that are either specified or measured, i.e.

$$
\begin{aligned}
& R_{1}=f_{R_{1}}\left(K, \omega_{p}, q_{p}, C_{3}, C_{4}, \beta\right) \\
& R_{2}=f_{R_{2}}\left(K, \omega_{p}, q_{p}, C_{3}, C_{4}, \beta\right) \\
& R_{4}=f_{R_{4}}\left(K, \omega_{p}, q_{p}, C_{3}, C_{4}, \beta\right)
\end{aligned}
$$

4) Trim resistors $R_{1}, R_{2}$ and $R_{4}$ to the values computed under 3 ).

At this point some comments seem in order.

The equations derived in step 1) and 3) are tuning equations and not design equations. The derivation of the design equations precedes the tuning process and determines the nominal value of each component including the gain $\beta$. Since there are more components than there are design equations, various optimization criteria can be taken into account in the design, such as minimum sensitivity, minimum gain-sensitivity product, ease of tuning, minimum component spread, upper and lower bounds on resistors and/or capacitors 


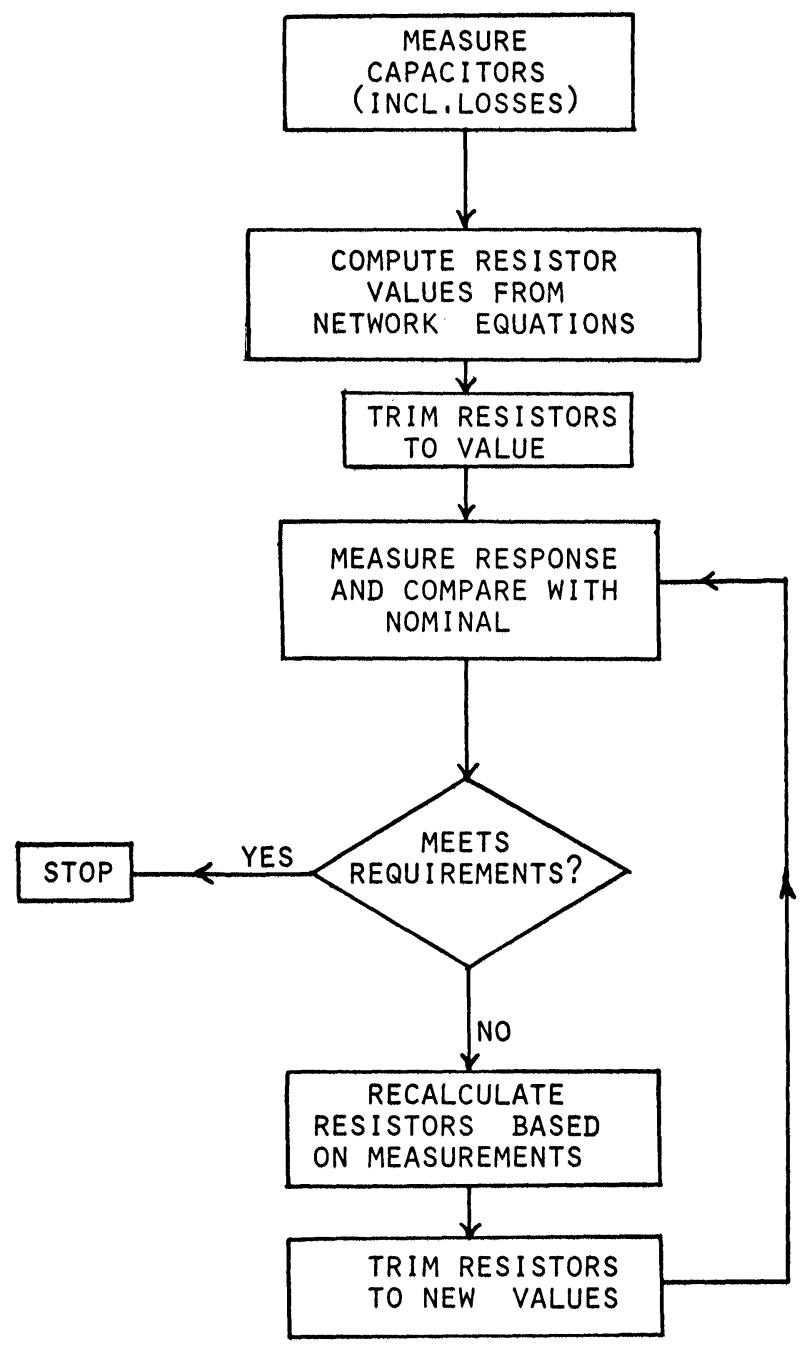

FIGURE 8 Deterministic tuning sequence. etc. In any event, the design, or nominal values of each component are known before the tuning process is begun. Thus, in the example of Figure 9, although the nominal value of $\beta$ is known, the actual value of $\beta$ obtained in manufacture is measured in step 2) by measuring resistors $R_{6}$ and $R_{7}$. (Note that at this point the amplifier is assumed to be ideal.)

For the Sallen-Key type circuits shown in Figures 5 and $9, \beta$ is not made equal to unity, but is larger (e.g. between one and two) if tight tolerances are specified for $q_{p}$. If necessary a final functional correction of $q_{p}$ can thereby be carried out without affecting the pole frequency $\omega_{p}$. Although this entails the inclusion of an additional resistor, namely $R_{7}$ ( $R_{6}$ is required to balance DC offset), this is a small price to pay compared with the increase in yield afforded by the possibility of a final touch-up tuning step. The deterioration of gain stability incurred by this slight increase in gain is negligible ${ }^{10}$ in hybridintegrated filters, due to the very close resistor tracking obtainable with either thick or thin film resistors. The combination of deterministic and functional tuning implied here will be further discussed under section 6 .

The measurement of the capacitors (i.e. $C_{3}$ and $C_{4}$ in step 2) above) should preferably be carried out after the (chip) capacitors have been assembled on the substrate. (In the case of thin film capacitors there is, of course, no other choice). This permits parasitic capacitance and assembly drift to be taken into account during the measurement. Although methods exist for the accurate measurement of individual capacitors when they are connected to additional circuitry $^{8}$, the preferred way of overcoming this problem is to provide provisional capacitor contacts to the substrate edge. These permit the measurement

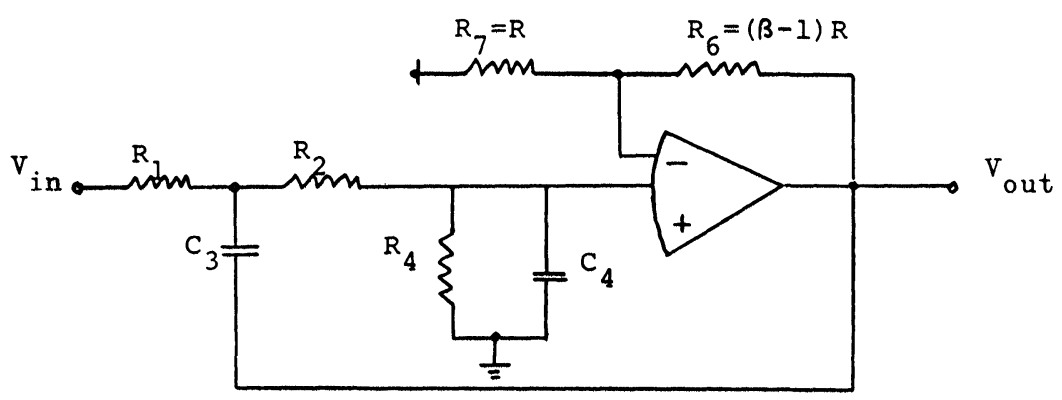

FIGURE 9 Second-order lowpass network. 


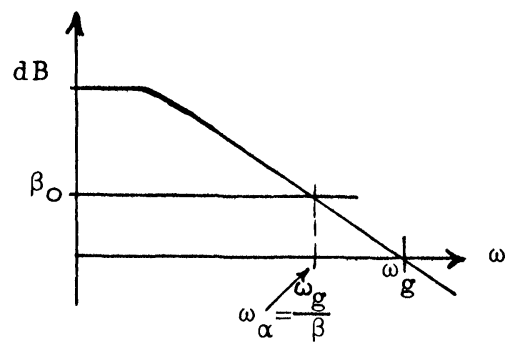

FIGURE 10 Frequency-dependent gain of an operational amplifier.

of either single capacitors, or combinations of parallel or series pairs, as required for the subsequent computations.

To attain a one-pass tuning procedure, parasitic effects due to nonideal circuit components must generally be taken into account in the network equations derived under step 3 above. This complicates the required computations considerably. The main parasitic effects that must be contended with are:

i) Non-ideal characteristics of the active devices (e.g. frequency dependent gain of the operational amplifiers). Referring to Figure 10 , this means that instead of using the constant gain $\beta_{0}$ in our equations, we must use $\beta(s)$, thus:

$$
\beta_{0} \rightarrow \beta(s) \approx \frac{\omega_{g}}{s+\omega_{\alpha}}
$$

ii) Losses and frequency dependence of capacitors. Thin-film capacitors, for example, are both lossy and frequency dependent. If the loss of a capacitor $C_{i}$ is $\tan \delta_{i}$, then, instead of $C_{i}$, we must approximate $C_{i}$ as follows:

$$
C_{i} \rightarrow \frac{C_{i}}{1+j \tan \delta_{i}} \approx \frac{C_{i}}{1+j \delta_{i}} \approx C_{i}\left(1-j \delta_{i}-\delta_{i}^{2}\right)
$$

Instead of frequency-independent capacitors, thinfilm capacitors will be frequency-dependent according to the relationship:

$$
C \rightarrow C(\omega)=C e^{-\omega \tau_{c}}
$$

whereby the time constant $\tau_{c}$ characterizes the technology used. Assuming that the value of $C(\omega)$ is measured at two frequencies (see Figure 11), the value of $C\left(\omega_{p}\right)$ can be obtained by extrapolation as follows:

$$
\log C\left(\omega_{p}\right)=\log C\left(\omega_{1}\right)+\tau_{c}\left(\omega_{p}-\omega_{1}\right)
$$

where

$$
\tau_{c}=\frac{\log C\left(\omega_{2}\right) / C\left(\omega_{1}\right)}{\omega_{2}-\omega_{1}}
$$

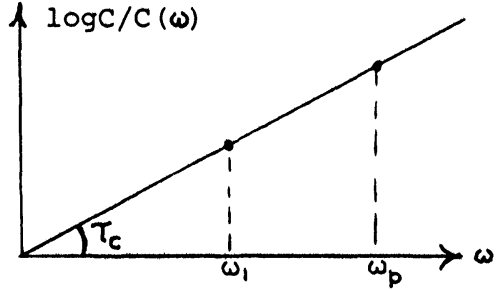

FIGURE 11 Frequency dependence of a thin-film capacitor.

iii) Parasitic capacitances on the circuit substrate and resistive losses along conductance paths.

These parasitics must be taken into account, to the extent that the response of the final assembled circuit is to be as accurate as if it had been tuned functionally. In doing so, the computations required under 3) above become rapidly more complex, the equations highly non-linear and of third, or even higher order. With increasing complexity, only numerical solutions by computer can be expected, whereas with ideal components, analytical solutions may be obtained. A more detailed discussion of the tuning of hybrid-integrated circuits with nonideal components is given elsewhere. ${ }^{8}$

\section{ACCURACY CONSIDERATIONS FOR DETERMINISTIC TUNING}

Using the deterministic tuning procedure, the accuracy attained depends on the accuracy with which capacitors can be measured, and resistors can be measured and trimmed. Assuming a worst-case measuring error of $\Delta C / C$ for all capacitors and an equal worst-case trimming error of $\Delta R / R$ for all resistors, we obtain a frequency error given by

$$
\left|\frac{\Delta \omega}{\omega}\right|_{\max } \leqslant\left|\frac{\Delta R}{R}\right|_{\max }+\left|\frac{\Delta C}{C}\right|_{\max }
$$

With a $0.1 \%$ accurate capacitance bridge and a capability of trimming and measuring resistors to within $0.05 \%$, the worst-case frequency error will be $0.15 \%$.

The accuracy with which the capacitor losses are measured will also effect the frequency accuracy, adding a term to the two in Eq. (24). Assuming a lossmeasurement error of $\Delta \delta$, we obtain the additional term:

$$
\frac{\Delta \omega}{\omega} \approx \frac{1}{2 q_{p}}\left(1+\frac{1}{4 q_{p}}\right) \Delta \delta
$$


This error is significantly smaller than the one represented by Eq. (24). Measuring the capacitor losses to within $10 \%$ accuracy (i.e. $\Delta \delta=0.1$ ) and assuming a pole $Q$ of only 2 (i.e. $q_{p}=2$ ), the frequency error given by Eq. (25) is $0.02 \%$.

In general, any function $F$ that is tuned to value using a resistor $R_{F}$ will be accurate to within $F(1 \pm \Delta F / F)$, where

$$
\frac{\Delta F}{F}=S_{R_{F}}^{F} \frac{\Delta R_{F}}{R_{F}}
$$

and $\Delta R_{F} / R_{F}$ is the trimming and measuring accuracy of the resistor $R_{F}$. Consider, for example, the case in which $q_{p}$ is to be tuned deterministically by adjusting the closed-loop gain $\beta$. This, in turn, is determined by a resistor ratio say $R_{\beta} / R$, where $R_{\beta}$ is the trimming resistor (e.g. $R_{6}$ or $R_{7}$ in Figure 9). The $q_{p}$ accuracy follows as

$$
\frac{\Delta q_{p}}{q_{p}}=S_{\beta}^{q} p S_{R_{\beta}}^{\beta} \frac{\Delta R_{\beta}}{R_{\beta}}
$$

For the network of Figure 9 Eq. (27) becomes:

$$
\frac{\Delta q_{p}}{q_{p}}=\left(1-\frac{1}{\beta}\right)\left(\frac{q_{p}}{\hat{q}}-1\right) \frac{\Delta R_{\beta}}{R_{\beta}}
$$

where $R_{\beta}=R_{6}$. If $R_{7}$ were used for $R_{\beta}$ the expression on the right-hand side of Eq. (28) would be negative. ${ }^{8}$ With a capability for measuring and trimming $R_{\beta}$ to within $0.05 \%$ accuracy, and with the typical values of $\beta=2, \hat{q}=0.4$ and $q_{p}=20$, we obtain $q_{p}$ accurately to within $1 \%$. Note that with currently available measuring equipment deterministic tuning can readily compete with functional tuning in terms of accuracy, provided that a sufficiently thorough computational effort and adequate computer facilities are invested in the process.

\section{COMBINING DETERMINISTIC WITH FUNCTIONAL TUNING}

Because deterministic tuning entails only component measurement, computation, and resistor trimming, it is the preferred tuning method, provided the production quantities are large enough to justify the initial computational effort (deriving the network equations including parasitic effects) and the cost of on-line computation facilities. Very often, the initial computational effort can be significantly reduced by deriving the equations of the idealized network and, after initial deterministic tuning, correcting the resulting error by a small number of functional tuning

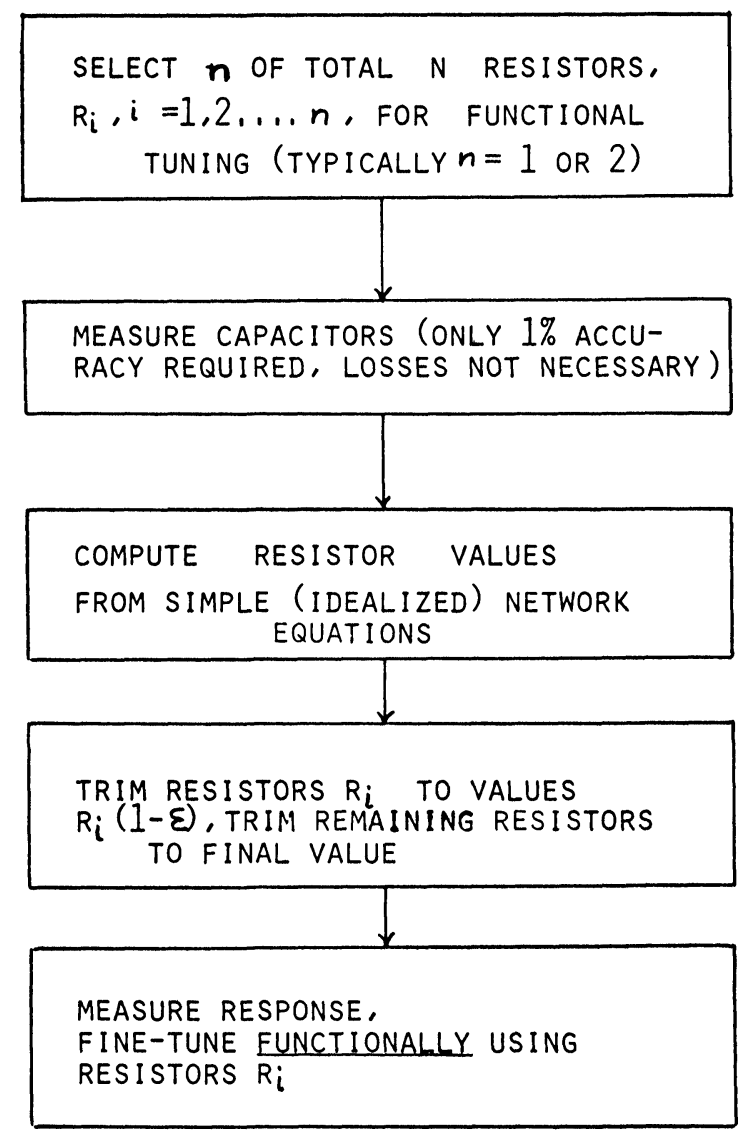

FIGURE 12 Combining deterministic with functional tuning.

steps. This procedure, outlined in Figure 12, eliminates the numerous and time-consuming iterative tuning steps generally required by the purely functional tuning procedure. It accomplishes this by reserving a small number (typically one or two) appropriate resistors for a final touch-up, or vernier functional adjustment after the circuit has previously been "coarse adjusted" deterministically using simple, i.e. idealized, network equations.

\section{SUMMARY}

The two main tuning procedures used for hybridintegrated active networks have been described. Their main characteristics are summarized in Table I.

Functional tuning is conceptually simple and very effective in that all stray and parasitic effects of a practical circuit can be tuned out "in situ" with the circuit in operation. The main disadvantages of the 
TABLE I

The main characteristics of functional and deterministic tuning

\begin{tabular}{|c|c|}
\hline Functional & Deterministic \\
\hline Network in operation & Network not in operation \\
\hline $\begin{array}{l}\text { Conceptually simple, directly } \\
\text { applicable }\end{array}$ & $\begin{array}{l}\text { Computationally complex, } \\
\text { requires considerable pre- } \\
\text { liminary analysis }\end{array}$ \\
\hline $\begin{array}{l}\text { Trim resistors to specified } \\
\text { function values (e.g. } \\
\text { amplitude, phase, frequency) }\end{array}$ & $\begin{array}{l}\text { Measure components and } \\
\text { compute resistor values, trim } \\
\text { resistors to computed values }\end{array}$ \\
\hline $\begin{array}{l}\text { Requires accurate phase and } \\
\text { frequency measurements }\end{array}$ & $\begin{array}{l}\text { Requires accurate resistor and } \\
\text { capacitor measurements }\end{array}$ \\
\hline $\begin{array}{l}\text { Tuning steps generally } \\
\text { interactive }\end{array}$ & $\begin{array}{l}\text { Resistor trimming non- } \\
\text { interactive }\end{array}$ \\
\hline Parasitic effects tuned out & $\begin{array}{l}\text { Parasitics included in network } \\
\text { equations }\end{array}$ \\
\hline Iterative (time consuming) & One-to-two trimming steps \\
\hline $\begin{array}{l}\text { Tuning sequence derivable } \\
\text { from sensitivity matrix }\end{array}$ & $\begin{array}{l}\text { Requires on-line computation } \\
\text { facilities and nonlinear } \\
\text { equation solving routines }\end{array}$ \\
\hline $\begin{array}{l}\text { Suitable for lab-purposes and } \\
\text { low production quantities }\end{array}$ & $\begin{array}{l}\text { Suitable for high production } \\
\text { quantities }\end{array}$ \\
\hline
\end{tabular}

method are the required time consuming iterations and the high-accuracy measurements of such parameters as phase, amplitude and frequency. Such measurements are often alien to the typical hybridcircuit manufacturing facility which is equipped for high-accuracy resistance and capacitance measurements only. Functional tuning thus becomes particularly useful for prototype and lab purposes and for low-quantity production. Deterministic tuning is based on the more sophisticated concept of predicting all relevant parasitic effects analytically and, by a combination of component measurements and computations of resistor values, trimming resistors to value such that the required network response is obtained. It is thereby a "one-shot" process in that, ideally, each resistor need be trimmed only once. The disadvantages of the method are the complexity of initial computation and the luxury of on-line computing facilities. Given sufficiently high production quantities, however, such initial outlays in brain and computer power may readily be justified and subsequently amortized. Finally, for the most common "intermediate" situation where production quantities are neither so high nor so low as to obviate the question of the most suitable tuning method, a combination of functional and deterministic tuning seems to offer the best results.

\section{REFERENCES}

1. G. S. Moschytz, "FEN Filter Design Using Tantalum and Silicon Integrated Circuits", Proc. IEEE, 58, Apr. 1970, pp. $550-566$.

2. J. Gensel, R. Meissner, V. Seidel, "An Active RC Filter for PCM Systems", Circuit Theory and Applications, 1, 1973.

3. W. B. Mikhael, L. K. Keys, "Standardizable Low-Pass Voice Band Hybrid Circuit Filters for PCM Communication Systems," IEEE Journ. Solid State Circuits, SC-10, 4, Aug. 1975, pp. 229-236.

4. W. Kraushar, U. Rickli, M. Schulthess, L. Süry, "MFC Filter", Hasler Mitteilungen, 3, Sept. 1975.

5. "Aktive Filter für PCM-Systeme in Dickschichttechnik", NTZ Kurier, NTZ 28 (1975), H.11.

6. R. Jeffers, D. G. Haigh, "Active RC Lowpass Filters for f.d.m. and p.c.m. Systems", Proc. IEE, 120, 9, Sept. 1973, pp. 945-953.

7. R. A. Friedenson, R. W. Daniels, R. J. Dow, P. H. McDonald, "RC Active Filters for the D3 Channel Bank", BSTJ, 54, 3, March 1975, pp. 507-529.

8. G. S. Moschytz, Linear Integrated Networks: Design, Van Nostrand Reinhold Co., New York, 1975, pp. 376-425; pp. 499-538.

9. G. S. Moschytz, Linear Integrated Networks: Fundamentals, Van Nostrand Reinhold Co., New York, 1974, p. 238.

10. G. S. Moschytz, "The Operational Amplifier in Linear Active Networks", IEEE Spectrum, 7, July 1970, pp. 42-50. 

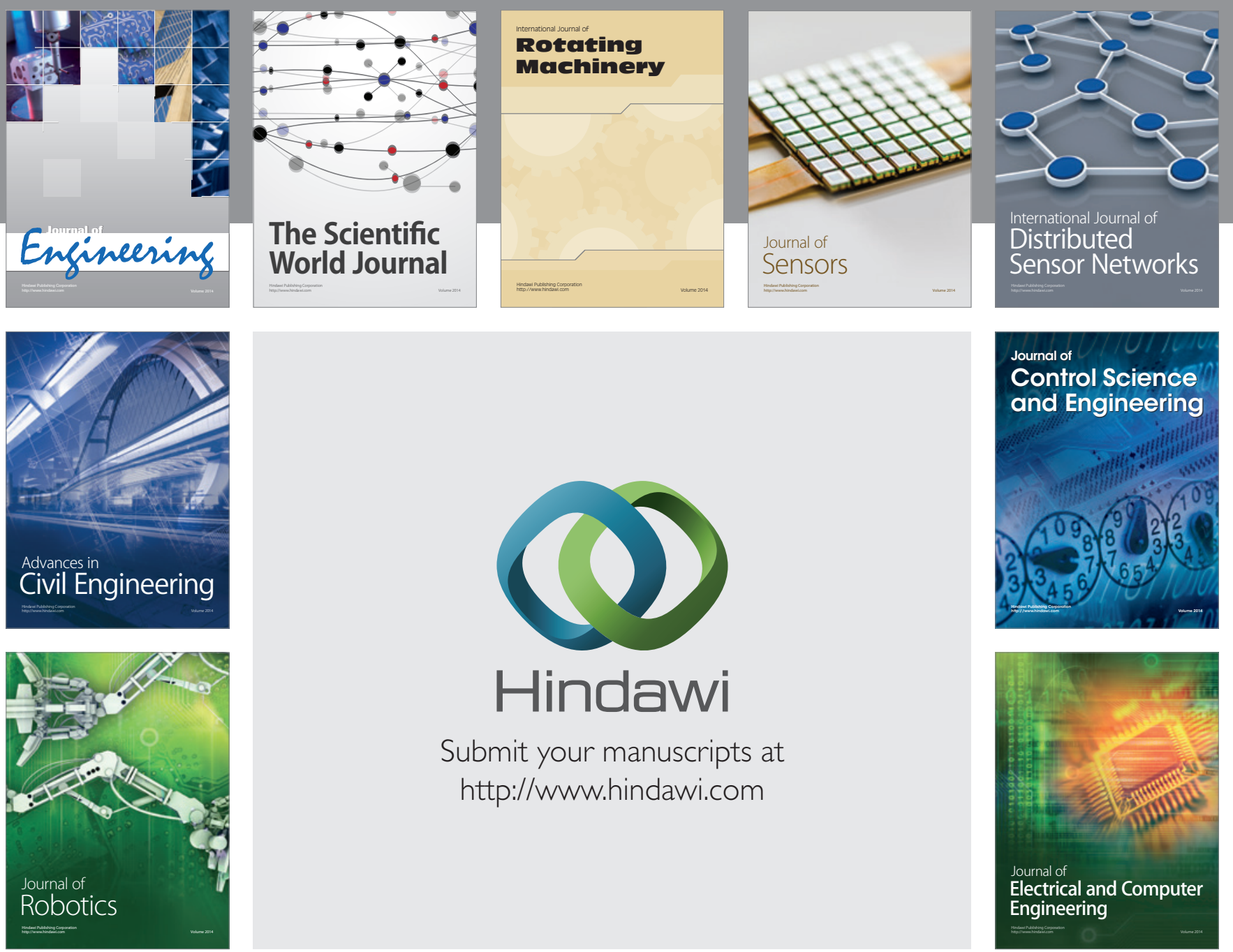

Submit your manuscripts at

http://www.hindawi.com
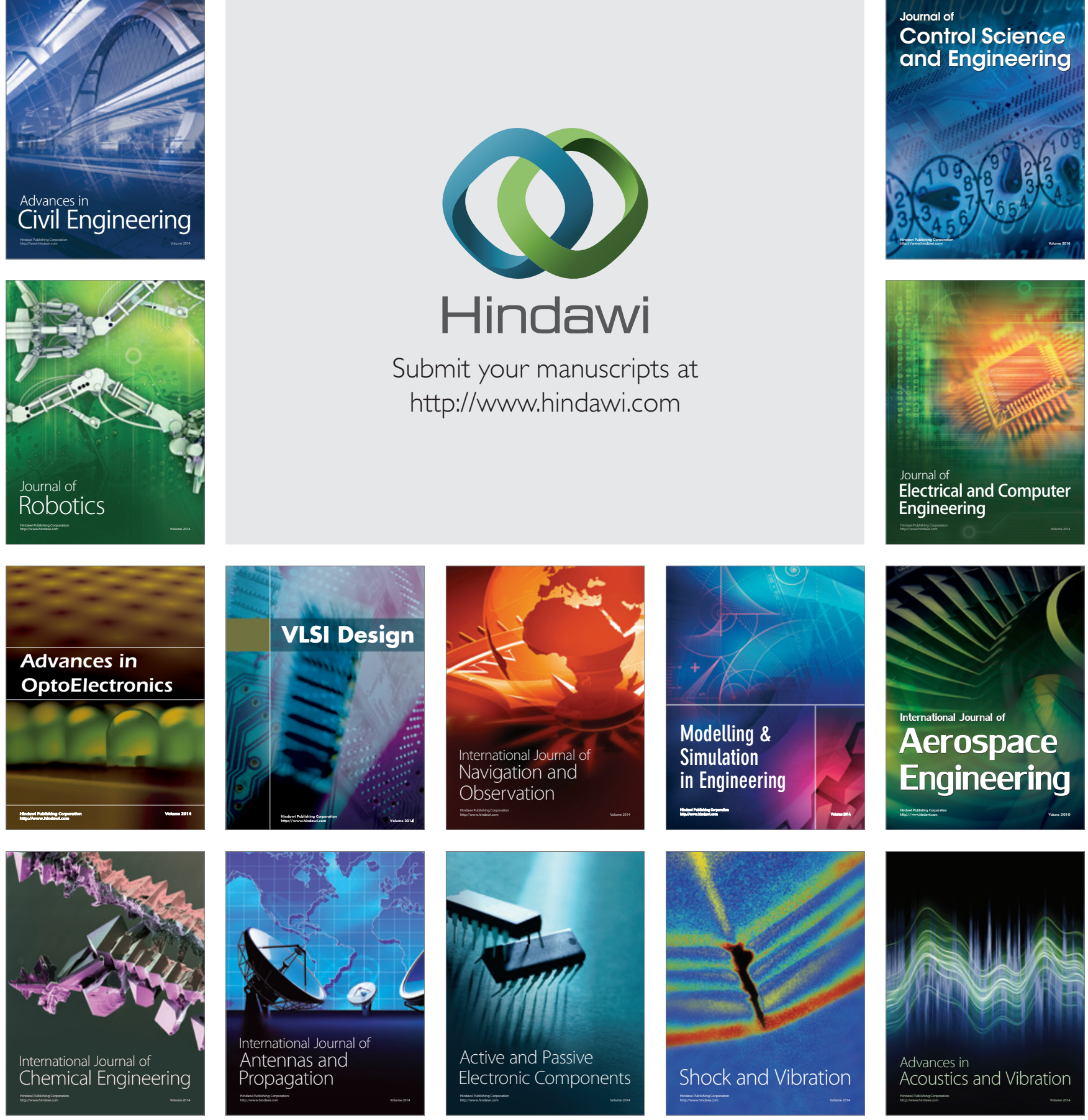